\title{
On Optimal and Cheat-Proof Packets Forwarding Strategies in Autonomous Ad Hoc Networks
}

\author{
Wei Yu and K. J. Ray Liu \\ Department of Electrical and Computer Engineering \\ University of Maryland, College Park, MD 20742 \\ Email: weiyu, kjrliu@isr.umd.edu
}

\begin{abstract}
This paper mathematically studies the cooperation and packet forwarding issues among selfish nodes in ad hoc networks under a game theoretic framework. Since in such packet forwarding games there usually exist an infinite number of Nash equilibria, a critical issue is how to perform equilibrium refinement, that is, how to apply extra optimality criteria to remove those equilibrium strategies that are less robust, less rational, or less likely. In this work, besides Pareto optimality and subgame perfection, other important optimality criteria, such as social welfare maximization, absolute fairness, and proportional fairness, have also been considered when performing equilibrium refinement. Combining with Pareto optimality and subgame perfection, each of these criteria can lead to a unique Nash equilibrium solution. Since nodes are selfish and will try to cheat whenever possible, the possible cheating behavior has also been fully exploited, and the analysis has shown that when cheating behaviors are considered, all these unique equilibrium solutions will converge to the same format, that is, a node should not help its opponent more than its opponent has helped it.
\end{abstract}

\section{INTRODUCTION}

A wireless ad hoc network is a group of (possibly mobile) nodes without requiring centralized administration or fixed network infrastructure, in which nodes can communicate with other nodes out of their direct transmission ranges through cooperatively forwarding packets for each other. Since ad hoc networks can be easily and inexpensively set up as needed, they have a wide range of applications. In many ad hoc networks, nodes may belong to different authorities and have different goals. Consequently, fully cooperative behaviors such as unconditionally forwarding packets for others cannot be taken for granted. On the contrary, in order to save limited resources, such as battery power, nodes may tend to be "selfish". We refer to such ad hoc networks as autonomous ad hoc networks.

In autonomous ad hoc networks, one major challenge is to stimulate cooperation among selfish nodes. In this paper we will investigate the cooperation issues in a game theoretic framework by mainly focusing on the most basic networking function, namely packet forwarding. Recently, some efforts have been made toward analyzing the cooperation in ad hoc networks in a game theoretic framework, such as [1]-[7]. In [1], Srinivasan et al. provide a mathematical framework for cooperation in ad hoc networks. In [3], Felegyhazi et al. identify the conditions under which cooperation strategies can

This work was supported in part by the Army Research Office under URI Award No. DAAD19-01-1-0494 form an equilibrium. In [4], Michiardi et al. study the cooperation among selfish nodes in a cooperative game theoretic framework. In [5], Altman et al. study the packet forwarding problem in a non-cooperative game theoretic framework. The study of selfish behaviors in ad hoc networks has also been addressed in [6], [7]. All of them use Nash equilibrium as optimality criterion.

However, there still exist some important issues that have not been fully addressed. First, there usually exist an infinite number of Nash equilibrium cooperation strategies, and in general not all of them are good. Therefore, one critical issue is how to remove those equilibria that are less robust, less rational, or less likely. This can be achieved by performing equilibrium refinement through introducing extra optimality criteria. In the literature, the following two extra criteria have been considered: Pareto optimality and subgame perfection [1], [5]. However, neither of them can reduce the solutions to a unique solution. To further remove those less robust, less rational, or less likely strategies, in this paper we have also introduced the following extra criteria: social welfare maximization, absolute fairness, and proportional fairness, and we have shown that when combining with Pareto optimality and subgame perfection, each of them can lead to a unique equilibrium cooperation strategy.

Second, the nodes' selfish nature has not been fully exploited in the existing works. In general, to maximize its own profit, a selfish node will not be willing to reveal its private information to others, and may even tend to cheat, such as reporting false information to others. However, some of the existing works, such as [1], assume that nodes will honestly report their private information to the others. Consequently, besides the above optimality criteria, cheat-proofing should also be adopted as the optimality criterion, that is, the cooperation strategy should guarantee that no node can increase its payoff through cheating. In this paper, the possible cheating behaviors have been fully exploited, and the analysis has shown that none of the obtained unique solutions are cheatproof. Further, the analysis has also shown that when the possible cheating behaviors are considered, all these unique solutions will converge to the same format, that is, a player should not help his opponent more than its opponent has helped him.

The rest is organized as follows. Section II studies a simple yet illustrating two-player packet forwarding game and the corresponding Nash equilibria. Section III investigates 
the equilibrium refinement under various optimality criteria. Section IV studies the cheat-proofing of the obtained equilibria and derives the optimal cooperation strategies. The related works are discussed in Section V. Finally Section VI concludes this paper.

\section{GAME MODEL}

In this section we focus on a simple yet illuminating twoplayer multi-stage packet forwarding game, which is modeled as follows. There are two players in this game, denoted by $N=\{1,2\}$. Each player needs its opponent to forward a certain number of packets in each stage. To simplify the illustration, we assume that all packets have the same size. For each player $i$, the cost to forward a packet is $c_{i}$, and the gain it can get for any packet that its opponent has forwarded for it is $g_{i}$. Here the cost can be the consumed energy and the gain is usually application-specific. Let $B_{i}$ be the number of packets that player $i$ will request its opponent to forward at each stage. The values of $B_{i}, c_{i}$, and $g_{i}$ will be reported by both players to each other, either honestly or dishonestly, before the game is started. It is also reasonable to assume that $g_{i} \geq c_{i}$, and there exists a $c_{\max }$ with $c_{i} \leq c_{\max }$.

Let $A_{i}=\left\{0,1, \ldots, B_{3-i}\right\}$ denote the set of actions that player $i$ can take in each stage, where $a_{i} \in A_{i}$ denotes that player $i$ will forward $a_{i}$ packets for its opponent in this state. We refer to an action profile $a=\left(a_{1}, a_{2}\right)$ as an outcome and denote the set $A_{1} \times A_{2}$ of outcomes by $A$. Then in each stage players' payoffs are calculated as follows provided the action profile $a$ being taken:

$$
\begin{aligned}
& u_{1}(a)=a_{2} \times g_{1}-a_{1} \times c_{1}, \\
& u_{2}(a)=a_{1} \times g_{2}-a_{2} \times c_{2} .
\end{aligned}
$$

That is, the payoff of a player is the difference between the total gain it obtained with the help of its opponent and the total cost it spent to help its opponent. We refer to $u(a)=$ $\left(u_{1}(a), u_{2}(a)\right)$ as the payoff profile associated with the action profile $a$. According to the backward induction principle [8], if this game will only be played for known finite times, the only Nash equilibrium (NE) is $a^{*}=(0,0)$, no matter whether the two players move simultaneously or not. That is, if the game will only be played for one time, no node will help its opponent. The same result also holds for the case when the stage game will be played for only finite times and the game termination time is known by both players.

Next we show that cooperation can still be achieved if the game will be played for infinite times, or for finite times but no player knows the exact game termination time. Let $G$ denote the repeated version of the above one-stage packet forwarding game. Let $s_{i}$ denote player $i$ 's behavior strategy, and let $s=\left(s_{1}, s_{2}\right)$ denote the strategy profile. Next we consider the following two utility functions:

$$
\begin{gathered}
U_{i}(s)=\lim _{T \rightarrow \infty} \frac{1}{T} \sum_{t=0}^{T} u_{i}(s), \\
U_{i}(s, \delta)=(1-\delta) \sum_{t=0}^{\infty} \delta^{t} u_{i}(s)
\end{gathered}
$$

Utility function (3) can be used when the game will be played for infinite times. The discounted version (4) can be used when the game will be played for finite times, but no one knows the exact termination time. Here the discount factor $\delta$ (with $0<\delta<1$ ) characterizes each player's expected playing time. Since in general the results obtained based on (3) can also be applied to the scenarios when (4) is used as long as $\delta$ approaches to 1 , in this section we will mainly focus on (3).

Now we analyze the possible NE for game $G$. According to the Folk theorem [8], for every feasible and enforceable payoff profile, there exists at least one NE to achieve it, where the set of feasible payoff profiles for the above game is

$$
V_{0}=\text { convex hull }\{v \mid \exists a \in A \text { with } u(a)=v\} .
$$

and the set of enforceable payoff profiles, denoted by $V_{1}$, is

$$
\begin{aligned}
V_{1}= & \left\{v \mid v \in V_{0} \text { and } \forall i: v_{i} \geq \underline{v}_{i},\right. \\
& \text { where } \left.\underline{v}_{i}=\min _{a_{-i} \in A_{-i}} \max _{a_{i} \in A_{i}} u_{i}\left(a_{-i}, a_{i}\right)\right\} .
\end{aligned}
$$

Fig. 1 depicts these sets for the game with $B_{1}=1$ and $B_{2}=$ 2 , where the vertical axis denotes player 1's payoff and the horizontal axis denotes player 2's payoff. The payoff profiles inside the convex hull of $\left\{(0,0),\left(g_{1},-c_{2}\right),\left(g_{1}-2 c_{1}, 2 g_{2}-\right.\right.$ $\left.\left.c_{2}\right),\left(-2 c_{1}, 2 g_{2}\right)\right\}$ (including the boundaries) are the set of feasible payoff profiles $V_{0}$, the set of payoff profiles inside the shading area (including the boundaries) are the set of feasible and enforceable payoff profiles $V_{1}$. We can easily check that as long as $g_{1} g_{2}>c_{1} c_{2}$, there exist an infinite number of NE. To simplify our illustration, in this paper, whenever introducing no ambiguity, we will use $x=\left(x_{1}, x_{2}\right)$ to denote the set of NE strategies corresponding to the enforceable payoff profile $\left(x_{2} g_{1}-x_{1} c_{1}, x_{1} g_{2}-x_{2} c_{2}\right)$.

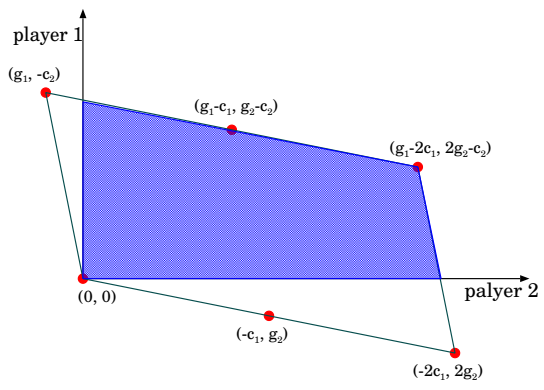

Fig. 1. Feasible and enforceable payoff profiles

\section{NASh EQUiLIBRIUM REFINEMENTS}

Based on the above analysis we can see that the infinitely repeated game $G$ may have an infinite number of NE. However, not all the obtained NE payoff profiles are simultaneously acceptable to both players. For example, the payoff profile $(0,0)$ will not be acceptable from both players' point of view if they are rational. Further, the existence of multiple NE payoff also requires nodes to make an agreement on which NE strategy should be used, which also introduces extra trouble. Next we show how to perform equilibrium refinement, that is, how to introduce new optimality criteria to eliminate those NE solutions which are less rational, less robust, or less likely. 
When performing equilibrium refinement, the following optimality criteria will be considered: Pareto optimality, subgame perfection, social welfare maximization, proportional fairness, and absolute fairness. In the literature, Pareto optimality has been used to refine the equilibrium in [1], and subgame perfection has been considered to remove empty threats in [6] However, as to be shown later, both are not able to reduce the solution set to a unique point, which is also one major motivation for us to introduce fairness constraint.

\section{A. Subgame Perfection}

Our first step towards refining the NE solutions is to rule out those empty threats. This motivates the equilibrium refinement based on more credible punishments known as subgame perfect equilibrium, which eliminates those equilibria in which the players' threats are empty. According to the perfect Folk theorem [8], every strictly enforceable payoff profile $v \in V_{2}$ is a subgame perfect equilibrium payoff profile of the game $G$, where

$$
\begin{aligned}
V_{2}= & \left\{v \mid v \in V_{0} \text { and } \forall i: v_{i}>\underline{v}_{i},\right. \\
& \text { where } \left.\underline{v}_{i}=\min _{a_{-i} \in A_{-i}} \max _{a_{i} \in A_{i}} u_{i}\left(a_{-i}, a_{i}\right)\right\} .
\end{aligned}
$$

That is, after applying the criterion of subgame perfection, only a small set of NE strategy profiles are removed.

\section{B. Pareto Optimality}

Our second step towards refining the set of NE solutions is to apply the criterion of Pareto optimality ${ }^{1}$. It is easy to check that only those payoff profiles lying on the boundary of the set $V_{0}$ could be Pareto optimal. Let $V_{3}$ denote the subset of feasible payoff profiles which are also Pareto optimal. For the case depicted in Fig. 1, $V_{3}$ is the set of payoff profiles which lie on the segment between $\left(g_{1},-c_{2}\right)$ and $\left(g_{1}-2 c_{1}, 2 g_{2}-c_{2}\right)$ and on the segment between $\left(g_{1}-2 c_{1}, 2 g_{2}-c_{2}\right)$ and $\left(-2 c_{1}, 2 g_{2}\right)$. After applying the criterion of Pareto optimality, a large portion of NE have been removed from the feasible set, but still there may exist an infinite number of NE strategy profiles. Let $V_{4}=V_{3} \cap V_{2}$ denote the set of payoff profiles which are both Pareto optimal and achievable through subgame perfect equilibria.

\section{Social Welfare Maximization}

Here the social welfare is referred to as the sum of the players' payoff, that is, $U_{1}(\delta, s)+U_{2}(\delta, s)$ when the strategy profile $s$ is used. Next we consider the equilibrium refinement based on the optimality criterion of maximizing the social welfare, that is,

$$
\arg \max _{s}\left(U_{1}(\delta, s)+U_{2}(\delta, s)\right) .
$$

Since in each stage $t$ we have

$$
u_{1}\left(a^{t}\right)+u_{2}\left(a^{t}\right)=a_{1}^{t}\left(g_{2}-c_{1}\right)+a_{2}^{t}\left(g_{1}-c_{2}\right),
$$

${ }^{1}$ Given a payoff profile $v \in V_{0}, v$ is said to be Pareto optimal if there is no $v^{\prime} \in V_{0}$ for which $v_{i}^{\prime}>v_{i}$ for all $i \in N$; $v$ is said to be strongly Pareto optimal if there is no $v^{\prime} \in V_{0}$ for which $v_{i}^{\prime} \geq v_{i}$ for all $i \in N$ and $v_{i}^{\prime}>v_{i}$ for some $i \in N$ [8]. then for each player $i$ its optimal strategy is always to forward all the packets that its opponent has requested him to forward if $g_{3-i}>c_{i}$ and refuse to forward any packet that its opponent has requested him to forward if $g_{3-i}<c_{i}$. That is, the optimal strategy is $x^{*}=\left(x_{1}^{*}, x_{2}^{*}\right)$ where

$$
\begin{array}{r}
x_{1}^{*}=\left\{\begin{array}{ccc}
B_{2} & \text { if } & g_{2}>c_{1} \\
\text { arbitrary } & \text { if } & g_{2}=c_{1} \\
0 & \text { if } & g_{2}<c_{1}
\end{array}\right. \\
x_{2}^{*}=\left\{\begin{array}{ccc}
B_{1} & \text { if } & g_{1}>c_{2} \\
\text { arbitrary } & \text { if } & g_{1}=c_{2} \\
0 & \text { if } & g_{1}<c_{2}
\end{array}\right.
\end{array}
$$

Here arbitrary means that arbitrary feasible value. It is easy to see that from the selfish nodes's point of view, many of the obtained payoff profiles are even not enforceable, for example, given $c_{i}$ and $g_{i}$, if $B_{1} \ll B_{2}$, we may have $U_{1}\left(\delta,\left(B_{2}, B_{1}\right)\right)<$ 0 . In another words, the goal of maximizing individual's payoff may conflict with the goal of maximizing the overall payoff. Since in many situations such criterion conflicts with the nodes' selfish nature, most likely it is not acceptable.

\section{Proportional Fairness}

Next we try to further refine the solution set based on the criterion of proportional fairness. Here a payoff profile is proportionally fair if $U_{1}(s) U_{2}(s)$ can be maximized, which can be achieved by maximizing $u_{1}(s) u_{2}(s)$ in each stage. Then we can reduce the solution set to a unique point as follows:

$$
x^{*}=\left\{\begin{array}{ccc}
\left(\frac{\frac{c_{2}}{g_{2}}+\frac{g_{1}}{c_{1}}}{2} B_{1}, B_{1}\right) & \text { if } & \frac{B_{1}}{B_{2}}<\frac{2}{\frac{c_{2}}{g_{2}}+\frac{g_{1}}{c_{1}}} \\
\left(B_{2}, B_{1}\right) & \text { if } & \frac{2}{\frac{c_{2}}{g_{2}}+\frac{g_{1}}{c_{1}} \leq \frac{B_{1}}{B_{2}} \leq \frac{\frac{c_{2}}{g_{1}}+\frac{g_{2}}{c_{2}}}{2}} \\
\left(B_{2}, \frac{\frac{c_{1}}{g_{1}}+\frac{g_{2}}{c_{2}}}{2} B_{2}\right) & \text { if } & \frac{B_{1}}{B_{2}}>\frac{\frac{c_{1}}{g_{1}}+\frac{g_{2}}{c_{2}}}{2}
\end{array}\right.
$$

\section{E. Absolute Fairness}

In many situations, absolute fairness may also be an important criterion. We first consider absolute fairness in payoff, which refers to that the payoff of these two players should be equal. By combining the criterion of Pareto optimality, the optimal strategy profile should be

$$
x^{*}=\left\{\begin{array}{lll}
\left(\frac{g_{1}+c_{2}}{g_{2}+c_{1}} B_{1}, B_{1}\right) & \text { if } \quad \frac{B_{1}}{B_{2}} \leq \frac{g_{2}+c_{1}}{g_{1}+c_{2}}, \\
\left(B_{2}, \frac{g_{2}+c_{1}}{g_{1}+c_{2}} B_{2}\right) & \text { if } \quad \frac{B_{1}}{B_{2}} \geq \frac{g_{2}+c_{1}}{g_{1}+c_{2}} .
\end{array}\right.
$$

Another similar criterion is absolute fairness in cost, which refers to that the cost spent by these two players for each other should be equal. By combining the criterion of Pareto optimality, the optimal strategy profile should be

$$
x^{*}=\left\{\begin{array}{lll}
\left(\frac{c_{2}}{c_{1}} B_{1}, B_{1}\right) & \text { if } \quad \frac{B_{1}}{B_{2}} \leq \frac{c_{1}}{c_{2}}, \\
\left(B_{2}, \frac{c_{1}}{c_{2}} B_{2}\right) & \text { if } \quad \frac{B_{1}}{B_{2}} \geq \frac{c_{1}}{c_{2}},
\end{array}\right.
$$

We can see that the obtained payoff profile is unique, feasible, and strictly enforceable. 


\section{Cheat-Proof Nash Equilibrium Strategies}

It is worth noting that the above unique solutions (12), (13) and (14) require players to reveal their private information to their opponents. While due to players' selfishness, it's unrealistic to expect them to honestly reveal their private information. Further, to maximize their own payoffs, selfish players may tend to cheat whenever they believe cheating can increase their payoffs. In this paper, we refer to a NE as cheat-proof if no player can further increase its payoff by revealing false private information to its opponents. After examining the three solutions (12), (13) and (14) (see below), it is surprising to see that none of them is cheat-proof. Since all these unique solutions are strongly Pareto optimal, the increase of its opponent's payoff will lead to the decrease of its own payoff. Therefore players have no incentive to honestly report their private information. On the contrary, they will cheat whenever cheating can increase their payoff.
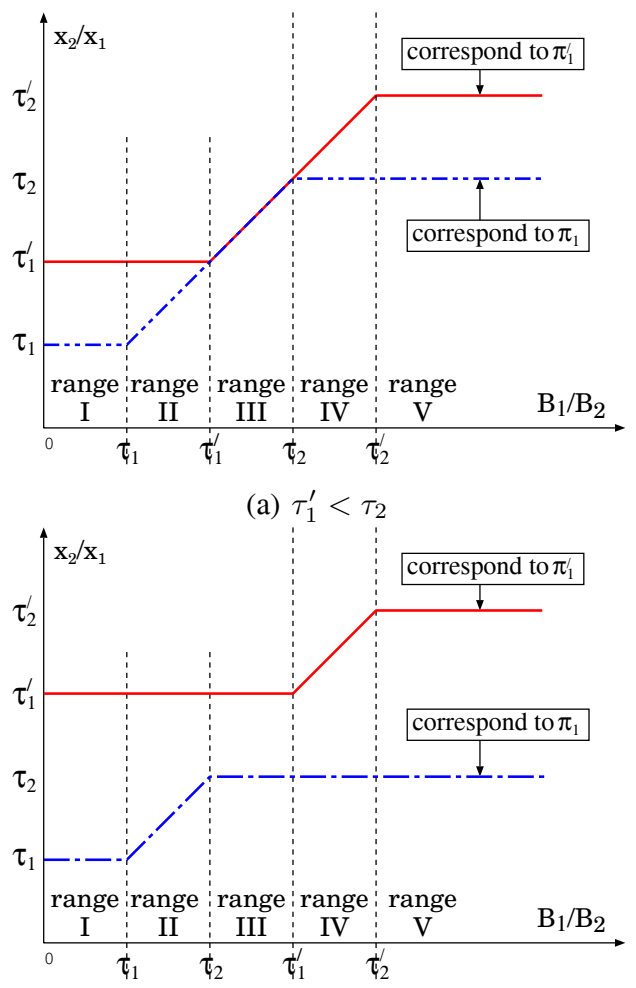

(b) $\tau_{1}^{\prime}>\tau_{2}$

Fig. 2. Player 1 falsely reports the value of $\pi_{1}$

We first study the solution (12). Let $\pi_{i}=c_{i} / g_{i}$ denote player $i$ 's cost-gain (CG) ratio. We first analyze whether player $i$ can increase its payoff by reporting a false CG ratio given that player 2 will honestly report its CG value. That is, we fix the value of $\pi_{2}$, let $\pi_{1}$ be player 1's true value, and let $\pi_{1}^{\prime}$ be the value that player 1 will falsely report with $\pi_{1}^{\prime}>\pi_{1}$. Let $\tau_{1}=\frac{2}{\pi_{2}+\frac{1}{\pi_{1}}}, \tau_{2}=\frac{\pi_{1}+\frac{1}{\pi_{2}}}{2}, \tau_{1}^{\prime}=\frac{2}{\pi_{2}+\frac{1}{\pi_{1}^{\prime}}}, \tau_{2}^{\prime}=\frac{\pi_{1}^{\prime}+\frac{1}{\pi_{2}}}{2}$. It is easy to check that $\tau_{1}<\tau_{1}^{\prime}$ and $\tau_{2}<\tau_{2}^{\prime}$. Recall that $B_{i}$ is the maximum number of packets that player $i$ will request its opponent to forward for it in each stage. Let $\left(x_{1}, x_{2}\right)$ denote the number of packets in average they will forward for each other in each stage according to the solution (12) given that the true values of $B_{1}$ and $B_{2}$ are known by both players. The relationship between $x_{2} / x_{1}$ and $B_{1} / B_{2}$ under different situations is illustrated in Fig. 2(a) and Fig. 2(b). In these two figures, the dashed curve corresponds to the relationship between $x_{2} / x_{1}$ and $B_{1} / B_{2}$ given that player 1 honestly reports its CG value, which is $\pi_{1}$; while the solid curve corresponds to the relationship between $x_{2} / x_{1}$ and $B_{1} / B_{2}$ given that player 1 falsely report its CG value, which is $\pi_{1}^{\prime}$.

From the results illustrated in Fig. 2 we can see that by falsely reporting a higher CG ratio, in most situations player 1 can increase the ratio of $x_{2} / x_{1}$. Next we study the effect of falsely reporting a high CG ratio on player 1's payoff. We first consider the situation that $\tau_{1}^{\prime} \leq \tau_{2}$, which is illustrated in Fig. 2(a). In this case the whole feasible space can be partitioned into 5 subareas along the feasible range of $B_{1} / B_{2}$ :

- For any value of $B_{1} / B_{2}$ inside range $\mathrm{I}$, the solution corresponding to $\pi_{1}$ is $\left(\frac{\pi_{2}+\frac{1}{\pi_{1}}}{2} B_{1}, B_{1}\right)$, and the solution corresponding to $\pi_{1}^{\prime}$ is $\left(\frac{\pi_{2}+\frac{1}{\pi_{1}^{\prime}}}{2} B_{1}, B_{1}\right)$. Since $\pi_{1}^{\prime}>\pi_{1}$, by falsely reporting a higher $\mathrm{CG}$ ratio player 1 can forward less packets for player 2 than it should, consequently increasing its own payoff.

- For any value of $B_{1} / B_{2}$ inside range II, the solution corresponding to $\pi_{1}$ is $\left(B_{2}, B_{1}\right)$, and the solution corresponding to $\pi_{1}^{\prime}$ is $\left(\frac{\pi_{2}+\frac{1}{\pi_{1}^{\prime}}}{2} B_{1}, B_{1}\right)$. Since $B_{2}>\frac{\pi_{2}+\frac{1}{\pi_{1}^{\prime}}}{2} B_{1}$, by falsely reporting a higher CG ratio player 1 can forward less packets for player 2 than it should, consequently increasing its own payoff.

- For any value of $B_{1} / B_{2}$ inside range III, the solution corresponding to $\pi_{1}$ is $\left(B_{2}, B_{1}\right)$, and the solution corresponding to $\pi_{1}^{\prime}$ is also $\left(B_{2}, B_{1}\right)$. That is, in this situation by changing the value of $\pi_{1}$ to $\pi_{1}^{\prime}$, player 1 's payoff will not change.

- For any value of $B_{1} / B_{2}$ inside range IV, the solution corresponding to $\pi_{1}$ is $\left(B_{2}, \frac{\pi_{1}+\frac{1}{\pi_{2}}}{2} B_{2}\right)$, and the solution corresponding to $\pi_{1}^{\prime}$ is $\left(B_{2}, B_{1}\right)$. Since $B_{1}>\frac{\pi_{1}+\frac{1}{\pi_{2}}}{2} B_{2}$, by falsely reporting a higher $C G$ ratio player 1 can request player 2 to forward more packets for it than player 2 should, consequently increasing its own payoff.

- For any value of $B_{1} / B_{2}$ inside range $\mathrm{V}$, the solution corresponding to $\pi_{1}$ is $\left(B_{2}, \frac{\pi_{1}+\frac{1}{\pi_{2}}}{2} B_{2}\right)$, and the solution corresponding to $\pi_{1}^{\prime}$ is $\left(B_{2}, \frac{\pi_{1}^{2}+\frac{1}{\pi_{2}}}{2} B_{2}\right)$. Since $\frac{\pi_{1}^{\prime}+\frac{1}{\pi_{2}}}{2} B_{2}>$ $\frac{\pi_{1}+\frac{1}{\pi_{2}}}{2} B_{2}$, by falsely reporting a higher CG ratio player 1 can request player 2 to forward more packets for it than player 2 should, and consequently increases its own payoff.

Similar results can also be obtained for the case that $\tau_{1}^{\prime}>$ $\tau_{2}$, where now player 1 can increase its own payoff over all possible values of $B_{1} / B_{2}$ by falsely reporting a higher $\pi_{1}$ value given that $\pi_{2}$ is fixed. In summary, by falsely reporting a higher $\pi_{1}$ value, in most situations player 1 can increase its payoff, and in no situations player 1's payoff will be decreased. Further, the higher player 1 reports the value of CG ratio, the 
more benefit player 1 can get. Similarly, player 2 can also increase its benefit by falsely reporting a higher CG ratio.

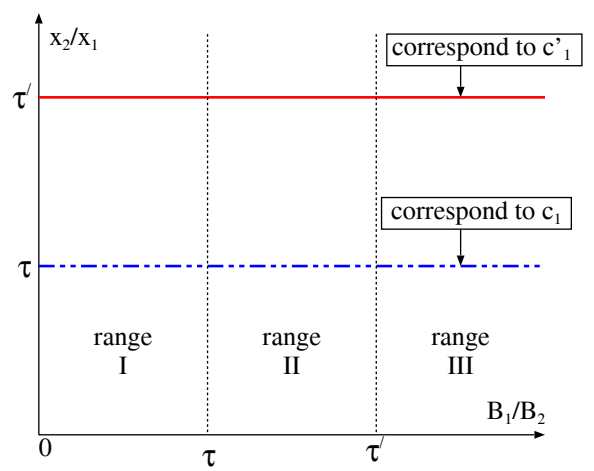

(a)

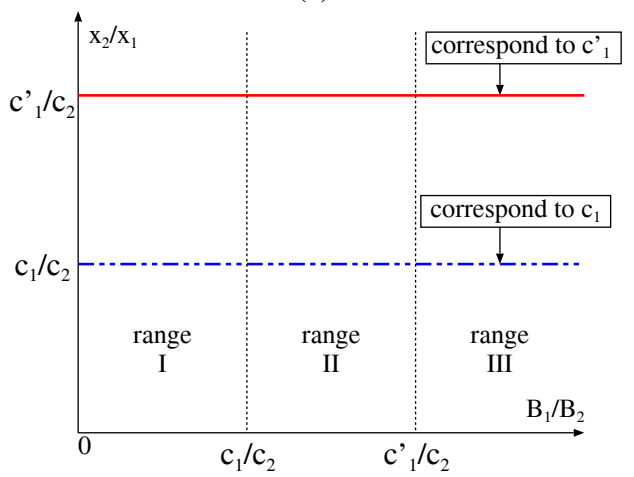

(b)

Fig. 3. Player 1 falsely reports its cost

Next we consider the solution (13). Now let $\tau=\frac{g_{2}+c_{1}}{g_{1}+c_{2}}$, and $\tau^{\prime}=\frac{g_{2}+c_{1}^{\prime}}{g_{1}+c_{2}}$, where $c_{1}<c_{1}^{\prime}$. Fig. 3(a) illustrates the relationship between $x_{2} / x_{1}$ and $B_{1} / B_{2}$ for the two different reported cost values $c_{1}$ and $c_{1}^{\prime}$, where $g_{1}, g_{2}$ and $c_{2}$ are fixed. Similar as in Fig. 2, the dashed curve corresponds to the case that player 1 reports a true cost value, while the solid curve corresponds to the case that player 1 reports a false cost value. From Fig. 3(a) we can see that by falsely reporting a higher cost value, in all situations player 1 can increase the ratio of $x_{2} / x_{1}$. Next we study the effect of falsely reporting a higher cost on player 1's payoff. As shown in Fig. 3(a), the whole space can be partitioned into 3 subareas along the feasible range of $B_{1} / B_{2}$ :

- For any value of $B_{1} / B_{2}$ inside range $\mathrm{I}$, the solution corresponding to $c_{1}$ is $\left(B_{1} / \tau, B_{1}\right)$, and the solution corresponding to $c_{1}^{\prime}$ is $\left(B_{1} / \tau^{\prime}, B_{1}\right)$. Since $\tau^{\prime}>\tau$, by falsely reporting a higher cost player 1 can forward less packets for player 2 than it should, then increase its own payoff.

- For any value of $B_{1} / B_{2}$ inside range II, the solution corresponding to $c_{1}$ is $\left(B_{2}, \tau B_{2}\right)$, and the solution corresponding to $c_{1}^{\prime}$ is $\left(B_{1} / \tau^{\prime}, B_{1}\right)$. Since $B_{1} / \tau^{\prime}<B_{2}$ and $B_{1}>\tau B_{2}$, by falsely reporting a higher cost, player 1 can forward less packets for player 2 than it should and request player 2 to forward more packets for it than player 2 should, then increase its own payoff.

- For any value of $B_{1} / B_{2}$ inside range III, the solution corresponding to $c_{1}$ is $\left(B_{2}, \tau B_{2}\right)$, and the solution corresponding to $c_{1}^{\prime}$ is $\left(B_{2}, \tau^{\prime} B_{2}\right)$. Since $\tau^{\prime}>\tau$, by falsely reporting a higher cost, player 1 can always request player 2 to forward more packets for it than player 2 should do, and consequently increase its own payoff.

In summary, by falsely reporting a higher $c_{1}$ value, in all situations player 1 can increase its payoff given that $c_{2}$ and $g_{2}$ are fixed. Further, the higher player 1 reports the value of $c_{1}$, the more benefit player 1 can get. Applying similar analysis it is also easy to show that by falsely reporting a lower $g_{1}$ value, in all situations player 1 can increase its payoff given that $c_{2}$ and $g_{2}$ are fixed. Similarly, player 2 can also increase its benefit by falsely reporting a higher $c_{2}$ or a lower $g_{2}$ given that $g_{1}$ and $c_{1}$ are fixed.

Now we consider the solution (14). Fig. 3(b) illustrates the relationship between $x_{2} / x_{1}$ and $B_{1} / B_{2}$ for the two different reported cost values $c_{1}$ and $c_{1}^{\prime}$ with $c_{1}^{\prime}>c_{1}$ and $c_{2}$ fixed. From Fig. 3(b) we can see that by falsely reporting a higher $c_{1}$ value, in all situations player 1 can increase the ratio of $x_{2} / x_{1}$. Applying similar analysis as before, we can conclude that given $c_{2}$ fixed, by falsely reporting a higher $c_{1}$ value, player 1 can always increase its payoff. Further, the higher player 1 reports the value of $c_{1}$, the more benefit player 1 can get. Similarly, player 2 can also increase its payoff by falsely reporting a higher $c_{2}$ value given $c_{1}$ fixed.

From the above analysis we can see that for any player $i$, honestly revealing its own private information such as $g_{i}$ and $c_{i}$ is always disadvantageous due to the reason that its opponent can utilize these information to get extra benefit. Since all the solutions we considered are (strongly) Pareto optimal solutions, the increase of its opponent's payoff usually leads to the decrease of its own payoff. So in the packet forwarding game with players being selfish, there is no incentive for the players to honestly reveal their private information. On the contrary, to maximize their own payoff, they will try to cheat whenever they believe that such cheating behaviors can increase their payoff.

What is the consequence if both players will cheat? Let's first examine the solution (12). In this case, based on the above analysis, both players will report a $c_{i} / g_{i}$ value as high as possible. Since we have assumed $g_{i} \geq c_{i}$ and $c_{i} \leq c_{\max }$, both player will set $g_{i}=c_{i}=c_{\max }$, and the solution (12) will become the following form:

$$
x^{*}=\left(\min \left(B_{1}, B_{2}\right), \min \left(B_{1}, B_{2}\right)\right) .
$$

After applying similar analysis for the solutions (13) and (14), it is surprising to see, but easy to understand, that both will also converge to the form (15). Accordingly, the corresponding payoff profile is

$$
v^{*}=\left(\left(g_{1}-c_{1}\right) \min \left\{B_{1}, B_{2}\right\},\left(g_{2}-c_{2}\right) \min \left\{B_{1}, B_{2}\right\}\right) .
$$

Besides $g_{i}$ and $c_{i}$, players can also report false $B_{i}$ information. However, it is easy to check that a rational player should always report a true $B_{i}$ value. Next we use player 
1 as an example to show this. If $B_{1} \leq B_{2}$, reporting a higher $B_{1}$ can only increase the number of packets it should forward for player 2, which introduces no gain to it, while by reporting a lower $B_{1}$, although it can decrease the number of packets forwarded for player 2, the number of its own packets forwarded by player 2 will also be decreased and cannot introduce gain to it too due to $g_{1} \geq c_{1}$. Similarly, if $B_{1}>B_{2}$, reporting a higher $B_{1}$ will not affect the solution, while reporting a lower $B_{1}$ may also reduce the number of packets that player 2 will forward for it, which introduces no gain as long as $g_{1} \geq c_{1}$. Therefore, player 1 should not report a false $B_{1}$ value. The same analysis also applies to player 2 .

In summary, when possible cheating behavior are considered, all the above solutions converge to the same form as in (15) with payoff being (16), that is, in the two-player packet forwarding game, in order to maximize its own payoff and be resistant to possible cheating behavior, a player should not forward more packets than its opponent does for it. A simple NE strategy to achieve the payoff profile (16) is as follows: For each player $i \in N$, in each stage $t$ it should forward $\min \left(B_{1}, B_{2}\right)$ packets for its opponent unless there was a previous stage $t^{\prime}$ in which its opponent has forwarded less than $\min \left(B_{1}, B_{2}\right)$ packets for it, in which case it chooses to stop forwarding packets for its opponent forever.

\section{Discussion}

The strategies proposed in [1], [6] may look similar to the one described above. In [1] Srinivasan et al. studied the cooperation in ad hoc networks by focusing on the energyefficient aspects of cooperation, where in their solution the nodes are classified into different energy classes and the behavior of each node depends on the energy classes of the participants of each connection. They have demonstrated that if two nodes belong to the same class, they should apply the same packet forwarding ratio. However, they require nodes to honestly report their classes, and a node can easily cheat to increase its own performance, such as the approach mentioned in [3] (Section VIII).

In [6], Urpi et al. claimed that it is not possible to force a node to forward more packets than it sends on average (Lemma 6.2), and then concluded that cooperation can be enforced in a mobile ad hoc network, provided that enough members of the network agree on it, and if no node has to forward more traffic that it generates (Theorem 6.3). However, the above analysis has shown that a strategy profile can still be enforceable even this may require a node to forward more packets than it sends on average, as illustrated in solutions (12), (13) and (14). Second, in mobile ad hoc network, due to the multihop nature, in general the number of packets a node forwards should be much more than the number of packets it generates. Accordingly, their strategy cannot enforce cooperation at all in most scenarios.

One major contribution of our analysis lies in that we have exploited all the possible NE strategies, demonstrated why some strategies are not good, why they cannot be acceptable by the players, and why the solution (15) is the only one that should be adopted. In other words, we have provided more insight and physical meaning for the solution (15).
The works presented in [9], [10] are also related to ours in the sense that cheating behavior has also been considered. They have proposed auction-based schemes to stimulate packet forwarding participation, where by using VCG-based second price auctioning these schemes force selfish nodes to honestly report their true private information (such as cost) to maximize their profit. However, in their schemes, a trusted third-party auctioneer is required per route selection and central banking services are needed to handle billing information, which usually cannot be satisfied in mobile ad hoc network. In our work, we focus on the scenario that neither trusted third-party auctioneer nor central banking service is available.

\section{CONCLUSION}

In this paper we have formally studied how to stimulate cooperation among selfish in autonomous ad hoc networks under a game theoretic framework. We have mainly focused on a simple yet illuminating two-player packet forwarding game. Since in such packet forwarding games there usually exist an infinite number of Nash equilibria, we have introduced various optimality criteria to further refine the obtained equilibria, which lead to the unique Nash equilibrium solutions which are more robust, more rational, and more likely. The following criteria have been considered when performing refinement: Pareto optimality, subgame perfection, social welfare maximization, absolute fairness, and proportional fairness. Since nodes are selfish and will try to cheat whenever possible, the possible cheating behavior has also been fully exploited. Our analysis has shown that when cheating behaviors are considered, all these unique equilibrium solutions will converge to the same format, that is, a selfish node should not help its opponent more than its opponent has helped it.

\section{REFERENCES}

[1] V. Srinivasan, P. Nuggehalli, C. F. Chiasserini, and R. R. Rao, "Cooperation in Wireless Ad Hoc Networks," in IEEE INFOCOM 2003.

[2] M. Felegyhazi, L. Buttyan, and J.-P. Hubaux, "Equilibrium Analysis of Packet Forwarding Strategies in Wireless Ad Hoc Networks - the Static case," Proceedings of Personal Wireless Communications, 2003.

[3] M. Felegyhazi, J.-P. Hubaux, and L. Buttyan, "Nash Equilibria of Packet Forwarding Strategies in Wireless Ad Hoc Networks," to appear in IEEE Transactions on Mobile Computing, 2005.

[4] P. Michiardi and R. Molva, "A Game Theoretical Approach to Evaluate Cooperation Enforcement Mechanisms in Mobile Ad hoc Networks," in WiOPT'03.

[5] E. Altman, A. A. Kherani, P. Michiardi, and R. Molva, "Non-cooperative Forwarding in Ad-Hoc Networks," Tech. Rep., INRIA, Sophia Antipolis, France, 2004.

[6] A. Urpi, M. Bonuccelli, and S. Giordano, "Modeling cooperation in mobile ad hoc networks: A formal description of selfishness," in WiOPT'03.

[7] J. Crowcroft, R. Gibbens, F. Kelly, and S. Ostring, "Modelling Incentives for Collaboration in Mobile Ad Hoc Networks," in WiOPT'03.

[8] M. J. Osborne and A. Rubinstein, A Course in Game Theory, The MIT Press, Cambridge, Massachusetts, 1994.

[9] L. Anderegg and S. Eidenbenz, "Ad Hoc-VCG: A truthful and costefficient routing protocol for mobile ad hoc networks with selfish agents," in ACM MobiCom 2003.

[10] S. Zhong, L. Li, Y. G. Liu, and Y. R. Yang, "On designing incentivecompatible routing and forwarding protocols in wireless ad-hoc networks - an integrated approach using game theoretical and cryptographic techniques," in ACM MobiCom, 2005, pp. 117-131. 